\title{
Infant rhesus macaques as a non-human primate model of Bordetella pertussis infection
}

Wenwen Jiang ${ }^{1,2}$, Chen Wei ${ }^{3}$, Dachao Mou ${ }^{1,2}$, Weilun Zuo ${ }^{1,2}$, Jiangli Liang ${ }^{1,2}$, Xiao Ma ${ }^{3}$, Lichan Wang ${ }^{3}, \mathrm{Na} \mathrm{Gao}^{1,2}$, Qin Gu ${ }^{1,2}$, Peng Luo ${ }^{3}$, Yan Ma ${ }^{1,2}$, Jingyan Li, ${ }^{1,2}$, Shuyuan Liu', Li Shi ${ }^{1 *}$ (D) and Mingbo Sun ${ }^{1,2^{*}}$

\begin{abstract}
Background: The prevalent resurgence of pertussis has recently become a critical public health problem worldwide. To understand pertussis pathogenesis and the host response to both the pathogen and vaccines, a suitable pertussis animal model, particularly a non-human primate model, is necessary. Recently, a non-human primate pertussis model was successfully established with baboons. Rhesus macaques have been shown to be ideal animal models for several infectious diseases, but a model of infectious pertussis has not been established in these organisms. Studies on rhesus macaque models of pertussis were performed in the 1920s-1930s, but limited experimental details are available. Recent monkey pertussis models have not been successful because the typical clinical symptoms and transmission have not been achieved.
\end{abstract}

Methods: In the present study, infant rhesus macaques were challenged with Bordetella pertussis (B.p) using an aerosol method to evaluate the feasibility of this system as an animal model of pertussis.

Results: Upon aerosol infection, monkeys infected with the recently clinically isolated B.p strain 2016-CY-41 developed the typical whooping cough, leukocytosis, bacteria-positive nasopharyngeal wash (NPW), and interanimal transmission of pertussis. Both systemic and mucosal humoral responses were induced by B.p.

Conclusion: These results demonstrate that a model of pertussis was successfully established in infant rhesus macaques. This model provides a valuable platform for research on pertussis pathogenesis and evaluation of vaccine candidates.

Keywords: Bordetella pertussis, Rhesus macaques, Infection, Transmission

\section{Background}

Pertussis is an acute respiratory disease caused mostly by the gram-negative bacterium Bordetella pertussis (B.p). The basic illness is non-inflammatory in nature and occurs without significant fever. The disease is characterized by non-productive paroxysmal coughs followed by periods of total respiratory normalcy, which makes it

\footnotetext{
* Correspondence: shili.imb@gmail.com; smb@imbcams.com.cn 1 Institute of Medical Biology, Chinese Academy of Medical Science \& Peking Union Medical College, Kunming 650118, Yunnan, China Full list of author information is available at the end of the article
}

different from all other infectious cough illnesses [1]. Severe respiratory failure is complicated by pulmonary hypertension, which may cause death, especially in infants [2]. Pertussis is transmitted directly from human to human, mostly via aerosolized respiratory droplets. Pertussis is a preventable disease, and its incidence decreased notably after vaccine immunization began. However, it has experienced a resurgence in several countries, even in countries with nearly universal vaccine coverage in the last 20 years [3-5]. A deeper understanding of the mechanism of pertussis pathogenesis and the

(C) The Author(s). 2021 Open Access This article is licensed under a Creative Commons Attribution 4.0 International License, which permits use, sharing, adaptation, distribution and reproduction in any medium or format, as long as you give appropriate credit to the original author(s) and the source, provide a link to the Creative Commons licence, and indicate if changes were made. The images or other third party material in this article are included in the article's Creative Commons licence, unless indicated otherwise in a credit line to the material. If material is not included in the article's Creative Commons licence and your intended use is not permitted by statutory regulation or exceeds the permitted use, you will need to obtain permission directly from the copyright holder. To view a copy of this licence, visit http://creativecommons.org/licenses/by/4.0/. The Creative Commons Public Domain Dedication waiver (http://creativecommons.org/publicdomain/zero/1.0/) applies to the data made available in this article, unless otherwise stated in a credit line to the data. 
host response to both the pathogen and the vaccines is urgently needed to enable this important public health concern to be faced. Therefore, it's urgent to develop suitable animal models for pertussis.

To establish animal models for pertussis, several studies have been carried out in mouse, rat, rabbit, and piglet models of pertussis [6, 7]. Unfortunately, these models have not been able to reproduce the full clinical spectrum observed in humans $[8,9]$. In studies using non-human primate (NHP) models, a baboon model has been successfully established [10]. Low-grade fever, paroxysmal coughing, leukocytosis, a long-lived antipertussis toxin (PT) antibody response, protection against subsequent challenge, and transmission have been achieved in this baboon model, which makes the model crucial for studies on the pertussis pathogenic mechanism as well as for the development of new vaccines and therapeutics [11]. Another NHP, the rhesus macaque, has been evaluated for use as a pertussis model since 1929, but none of the studies have been able to completely replicate the human clinical disease [1214]. In contrast, 2 studies using Macaca (M.) cyclopis have investigated the similarity of the disease in these organisms to the human pertussis clinical syndrome [15, 16]. Baboons and macaques are Old World monkeys that were separated approximately 10 million years ago, and rhesus macaques are closely related to M. cyclopis. Several disease models have been established using macaques and/or baboons. While baboon models suffer from limited availability, high housing costs, and a lack of suitable reagents for use in these monkeys, rhesus macaques are more readily available and have low housing costs, and suitable reagents are available [17].

Therefore, we infected rhesus macaques with B.p via aerosol challenge. We investigated clinical symptoms, including leukocytosis, coughing, and nasopharyngeal colonization; analysed the humoral and mucosal immune response and cytokine levels; and performed a transmission test to evaluate the suitability of infant rhesus macaques as a potential alternative NHP model for pertussis.

\section{Methods}

\section{Animals}

The infant rhesus macaques used in this study (5-6 months of age) were obtained from the Institute of Medical Biology, Chinese Academy of Medical Sciences (IMBCAMS). The study protocol was approved (DWSP201809002) by the Committee on Ethics of the IMBCAMS, and the study was conducted in strict accordance with the Guidelines for the Care and Use of Laboratory Animals published by the National Research Council of the National Academies and the Guidance for Experimental Animal Welfare and Ethical Treatment published by the Ministry of Science and Technology of the People's Republic of China (2006). During the study periods, the monkeys were maintained at Animal Biosafety Level 2, housed individually in cages in a climatecontrolled room (temperature of $18-25^{\circ} \mathrm{C}$ and humidity $30-70 \%$ ) with a $12 \mathrm{~h}$ light/dark cycle, given chow and fruits in strictly accordance with the animal welfare requirements and allowed free access to water. After the experiment, the monkeys were confirmed to have completely recovered from B.p infection and were returned to the Monkey Mountain of IMBCAMS, where they were allowed to live until they died naturally.

\section{Bacterial strains and media}

The B.p strain 2016-CY-41 used in this study was recently isolated from a patient in China and was obtained from the National Institutes for Food and Drug Control (Beijing, China). The polymorphisms in the PT promoter (ptxP), PT subunit 1 (ptxA), pertactin (prn), fimbrial (fim) 2 and fim3 were assessed by DNA sequencing. The genotype of 2016-CY-41 was ptxP1/ptxA1/prn1/fim2-1/ fim3-1. For B.p infection experiments, bacteria were grown on Bordet-Gengou agar (B-G) plates (BG, Hopebio, CHN) containing 20\% defibrinated sheep blood (Nanjinglezhen, $\mathrm{CHN}$ ) for 48 to $72 \mathrm{~h}$ at $37^{\circ} \mathrm{C}$. Colonies from fresh $\mathrm{B}-\mathrm{G}$ plates were resuspended in isotonic saline, diluted to a concentration of $10^{11} \mathrm{CFU} / \mathrm{mL}$ using a turbidimetric method, and used within $2 \mathrm{~h}$ of preparation. For culture of nasopharyngeal wash (NPW) bacteria, Regan-Lowe plates prepared from Regan-Lowe charcoal agar base with $10 \%$ defibrinated sheep blood and $40 \mu \mathrm{g} / \mathrm{mL}$ cephalexin (Oxoid, US) were used.

\section{Infection and transmission in rhesus macaques}

Seven healthy male rhesus macaques, aged 5 to 6 months and weighing $1.2-1.8 \mathrm{~kg}$, were obtained from the IMBCAMS (Animal Licence No. SCXK (Dian) K20150004). Before the experiment started, immunoglobulin G (IgG) against filamentous haemagglutinin (FHA) was detected to confirm that the monkeys were negative for infections of B.p, B. parapertussis, and B. bronchiseptica. Then, the seven animals were randomly assigned to two groups (Table 1). Group 1, containing 5 macaques, was challenged with strain 2016-CY-41 via aerosol exposure using an aerosolization apparatus designed by our laboratory and produced by Lanfang Honlan Equipment Co (Additional file 1). The apparatus was composed of a rectangular Plexiglas chamber with a removable lid (40 $\mathrm{cm} \times 60 \mathrm{~cm} \times 40 \mathrm{~cm}$ ), a pump and a medical nebulizer (average atomization rate: $\geq 0.15 \mathrm{~mL} / \mathrm{min}$, working pressure: $60-150 \mathrm{kPa}$, normal working temperature: 10 $40{ }^{\circ} \mathrm{C}$ ). The pump was connected to the inlet side of the nebulizer to deliver a B.p suspension for atomization. The outlet side of the nebulizer was connected to two 
Table 1 Experimental grouping for Bordetella pertussis infection in rhesus macaques

\begin{tabular}{|c|c|c|c|c|c|c|}
\hline Group & Monkey ID & Sex & Age (month) & Weight (kg) & B.p Strain & Infection route \\
\hline \multirow[t]{5}{*}{ Group 1} & 18,089 & $M$ & 6 & 1.5 & $2016-C Y-41$ & Aerosol challenge \\
\hline & 18,105 & M & 6 & 1.8 & 2016-CY-41 & Aerosol challenge \\
\hline & 18,043 & M & 5 & 1.1 & 2016-CY-41 & Aerosol challenge \\
\hline & 18,053 & M & 5 & 1.2 & 2016-CY-41 & Aerosol challenge \\
\hline & 18,093 & M & 6 & 1.5 & 2016-CY-41 & Aerosol challenge \\
\hline \multirow[t]{2}{*}{ Group 2} & 18,073 & M & 6 & 1.5 & $2016-C Y-41$ & Transmission \\
\hline & 18,107 & M & 5 & 1.2 & 2016-CY-41 & Transmission \\
\hline
\end{tabular}

inlet ports of the challenge chamber to deliver atomized B.p to the interior of the chamber. An outlet tube with an air filter was connected to the challenge chamber to remove air. An air sampling port was embedded in the middle of the challenge chamber to monitor the actual concentration of aerosolized B.p inside the chamber. Animals were infected via the challenge chamber for 60 min. Within the $60 \mathrm{~min}$ period, the air sample was removed from the sampling port every $10 \mathrm{~min}$ for assessment of the concentration of B.p inside the chamber.

At 2 days post infection (dpi), 1 challenged macaque was cohoused with 1 naive animal in one cage, and the animals were separated after 4 days to investigate transmission. The 2 macaques that were cohoused with 2016CY-41-challenged animals formed group 2.

\section{Animal evaluation and sample collection}

A schematic of the specimen collection timeline is displayed in Fig. 1. Total white blood cell (WBC) counts were measured by blood cell counting. Coughing frequency was monitored with a recording device. The data were reviewed, and the numbers of coughs during four 30-min periods each day (7:00-7:30 a.m., 10:00-10: 30 a.m., 2:00-2:30 p.m., and 8:00-8:30 p.m.) were calculated. The average number of coughs per hour for each day was calculated as the mean for all four observation periods for all animals in each group. For NPW collection, animals were anaesthetized using ketamine hydrochloride $(10 \mathrm{mg} / \mathrm{kg})$. A piece of tubing approximately 15 $\mathrm{cm}$ in length and $0.6 \mathrm{~mm}$ in diameter was slowly inserted into the back of the nostril. A syringe connected to the end of the tubing was used to slowly inject $1 \mathrm{~mL}$ of PBS into the nostril, and fluid was collected in a sterile dry container. This process was repeated for the other nostril, and fluid was collected into the same container as that used for the first nostril. The NPW was serially diluted in saline and plated on Regan-Lowe plates. The number of CFUs was calculated after 4-5 days of incubation at $37^{\circ} \mathrm{C}$. The B.p colonies were identified by examining colony morphology and haemolysis on

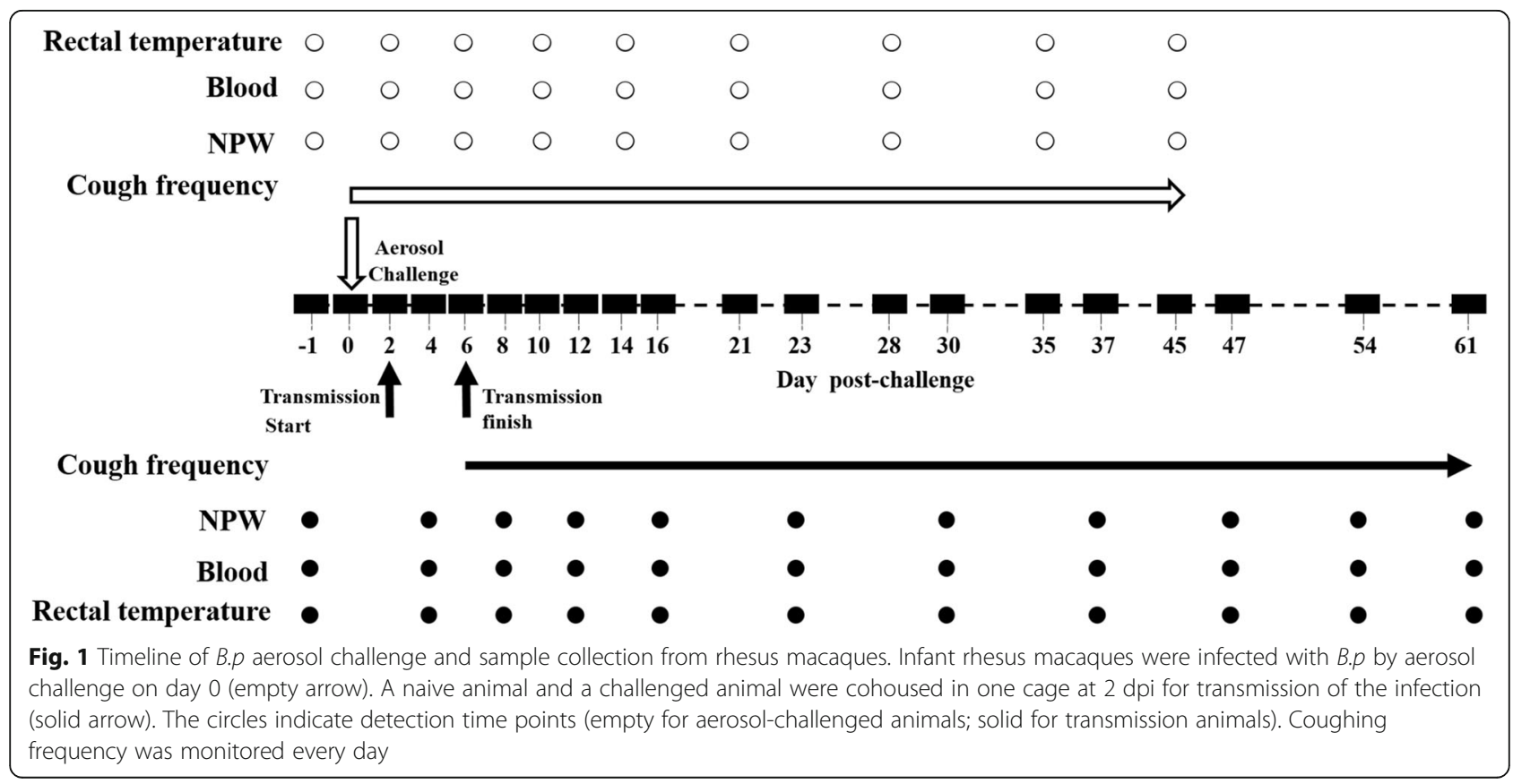


Regan-Lowe plates and by polymerase chain reaction (PCR) amplification of IS481, a genomic insertion site that is specific for B.p [18].

\section{Detection of antibodies in serum and NPW}

Serum and NPW were collected, and anti-PT, anti-FHA, anti-PRN and/or anti-adenylate cyclase toxin (ACT) IgG and/or IgA levels were measured using an enzymelinked immunosorbent assay (ELISA). Microplates (96well) were coated with the antigens PT, FHA, PRN, and/ or ACT at $3 \mu \mathrm{g} / \mathrm{mL}$ and incubated at $4{ }^{\circ} \mathrm{C}$ overnight. Then, the plates were blocked with $3 \%(\mathrm{w} / \mathrm{v})$ bovine serum albumin (BSA, Amresco, A0332) in phosphatebuffered saline (PBS) at $37^{\circ} \mathrm{C}$ for $2 \mathrm{~h}$. Diluted serum or NPW was added to each microplate and incubated at $37^{\circ} \mathrm{C}$ for $1 \mathrm{~h}$. After washing, horseradish peroxidase (HRP)-labelled sheep anti-monkey IgG (Invitrogen, USA, PA1-84631) or anti-human IgA (Jackson ImmunoResearch Laboratories, USA, 109-036-011) was added to the microplate, and the plate was incubated at $37^{\circ} \mathrm{C}$ for $1 \mathrm{~h}$. All of the ELISA plates were developed using tetramethylbenzidine (TMB; Solarbio, CHN, PR1200) to generate a colorimetric reaction, and the reaction was terminated with $2 \mathrm{~mol} / \mathrm{L} \mathrm{H}_{2} \mathrm{SO}_{4}$. For each set of ELISA plates subjected to IgG detection, a pertussis antiserum WHO international standard was used as a reference (NIBSC code: 06/140). For assessment of anti-ACT IgG in serum and antibody responses in NPW, a blank sample was included on each plate, and an optical density (OD) values $\geq 2.1$-fold that of the blank sample was set as the cut-off value (all the antigens were from the Department of DTP Vaccine and Toxin, National Institute for Food and Drug Control, China). The results are presented as geometric mean concentrations (GMCs) or geometric mean titres (GMTs) and their 95\% confidence intervals (CIs).

\section{Measurement of cytokines}

Serum concentrations of interleukin (IL)-1 $\beta$, IL-4, IL-6, IL-8, IL-10, IL-12/23p40, IL-13, IL-17A, interferon (IFN) $-\gamma$, and tumour necrosis factor (TNF)- $\alpha$ were detected by the Luminex technique with a MILLIPLEX NHP Magnetic Bead Panel (Merck Millipore, US) according to the manufacturer's instructions. An unpaired t-test was used to test for differences between the prechallenge cytokine production and the peak cytokine production during the post-challenge period $(2 / 4,6 / 8$, $10 / 12,14 / 16,21 / 23$, and $28 / 30 \mathrm{dpi}$; the latter is for the transmission group, as indicated in Fig. 1) for each animal due to the highly variable starting concentrations between animals and the variability of the peak response for each cytokine post infection.

\section{Statistics}

The data were graphed and analysed using GraphPad Prism version 7.0 (GraphPad Software, Inc.). The data are presented as the means \pm standard errors of the means or as the GMCs/GMTs and their 95\% CIs. Unpaired Student's t-test was utilized to assess statistical significance.

\section{Results}

Clinical signs in infant rhesus macaques after B.p infection The concentration of bacteria in the challenge chamber reached and was maintained at $10^{4}-10^{5} \mathrm{CFU} / \mathrm{mL}$. In the challenged group, all 5 animals developed classic symptoms of clinical pertussis. The number of WBCs was significantly increased 2- to 5 -fold beginning at $6 \mathrm{dpi}$, reached the highest level at $14 \mathrm{dpi}$, and returned to baseline by 28 dpi (Fig. 2a). The number of bacterial colonies from the NPW increased from $2 \mathrm{dpi}$ and reached the highest level, $6.2 \times 10^{6} \mathrm{CFU} / \mathrm{mL}$, at $10 \mathrm{dpi}$; then, the number of colonies gradually decreased until approximately 45 dpi (Fig. 2b). In addition, all animals developed severe coughs that persisted for over 4 weeks. In the early stage after challenge, the animals developed a mild cough. At $10 \mathrm{dpi}$, the cough seemed to worsen, especially at night (Fig. 2c). At peak illness, the cough became violent, lasting 10-20 s (Additional file 2). However, the rectal temperature was not significantly different from that in the pre-challenge period and was maintained between $37.2^{\circ} \mathrm{C}$ and $39.9^{\circ} \mathrm{C}$ (Additional file 3).

\section{Antibody response in the challenged group}

Production of IgG antibodies against PT, FHA, PRN, and ACT was induced in all 5 macaques in the challenged group. The seroconversion rate reached $100 \%$ at day 14 for anti-PT and anti-FHA. For anti-PRN, the seroconversion rate reached $100 \%$ at day 35 with a slight delay, but it reached $60 \%$ at day 14 and remained at $80 \%$ from day 28 onwards during the investigation period. The levels of anti-PT, anti-FHA and anti-PRN antibodies significantly increased from 14days onwards and reached approximately 200 -fold, 22 -fold, and 11 -fold on day 35 , respectively. In addition, they remained at high levels with GMCs of $598.9 \mathrm{IU} / \mathrm{mL}$ (95\% CI, 559.7-640.8), $112.0 \mathrm{IU} / \mathrm{mL} \quad(95 \% \mathrm{CI}, 84.11-149.2)$ and $9.8 \mathrm{IU} / \mathrm{mL}$ (2.08-45.88) on day 45, respectively (Fig. 3a-c). The anti-ACT levels were significantly elevated from day 14 onwards and remained stable with GMTs of 9.79 (95\% CI, 7.84-12.23), 10.82 (95\% CI, 8.91-13.14), and 10.68 (95\% CI, 8.96-12.74), respectively, on day 28,35 , and 45 (Fig. 3c).

Furthermore, both IgA and IgG antibodies against PT, FHA, and PRN in NPW were assessed in all 5 animals in the challenged group at the indicated times. The levels 

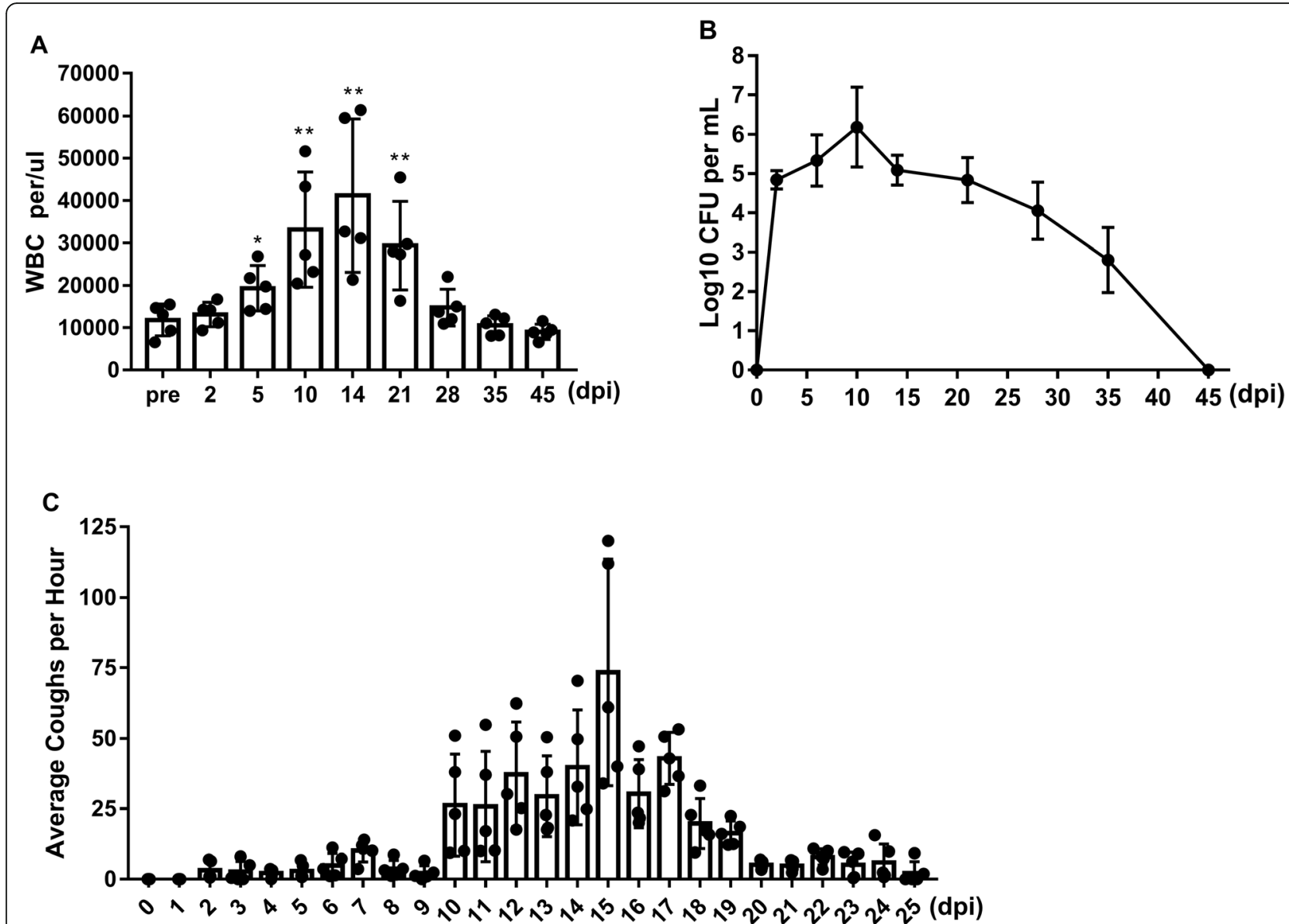

Fig. 2 Leukocytosis, B.p colonization, and coughing in B.p-infected rhesus macaques. a Dynamic profiles of the number of WBCs per $\mu L$ of peripheral blood in B.p-infected animals. $\mathbf{b}$ The CFUs per $50 \mu \mathrm{L}$ of NPW in B.p-infected animals were recorded. $\mathbf{c}$ The number of coughs per day was recorded for the B.p s-infected group. ND, no data. ${ }^{*}, P<0.01 ;{ }^{*}, P<0.005$. The bars with error bars represent the means $\pm S D s(n=5)$

of IgA against PT started to rise at $28 \mathrm{dpi}$ and reached a maximum at $35 \mathrm{dpi}$; the levels of IgA against FHA were also significantly increased at $28 \mathrm{dpi}$ and continued to rise at $45 \mathrm{dpi}$ (Fig. 4a-b). However, no increases in the levels of IgA against PRN were observed in these monkeys. A previous study has suggested that human NPW contains IgG that probably enters from the circulation via transudation [19]; therefore, IgG antibodies in NPW were also evaluated. The specific IgG antibody levels in NPW at pre-challenge were very low. Anti-PT IgG levels were significantly increased in all five animals at $21 \mathrm{dpi}$ and peaked at $28 \mathrm{dpi}$ (Fig. 4c). Anti-FHA IgG levels rose in all five animals starting at $14 \mathrm{dpi}$ and continued to increase at $45 \mathrm{dpi}$ (Fig. 4d). However, not all animals showed increases in anti-PRN IgG titres after challenge (Fig. 4e).

\section{Transmission}

Four days after separation from the 2016-CY-41-challenged macaques, both macaques in group 2 became infected, as demonstrated by prominent leukocytosis, with a peak level between 2- and 4-fold greater than the preinfection level (Fig. 5a). In addition, B.p was recovered from the NPW, with the highest numbers reaching $5.4 \times$ $10^{6}$ and $7.0 \times 10^{6} \mathrm{CFU} / \mathrm{mL}$ (Fig. 5b). More importantly, both animals also developed severe coughs (Fig. 5c). Antibody responses to B.p were also observed and exhibited trends similar to that seen following primary infection in group 1 (Fig. $5 \mathrm{~d}-\mathrm{g}$ ).

We also measured the levels of IgA and IgG antibodies against PT, FHA, and PRN in the NPW of the two transmission animals. IgA against PT and FHA was observed (Fig. 6a-b), and the levels of IgG against PT and FHA were also markedly increased (Fig. 6c-d). However, only a low level of anti-PRN IgG was detected for a very short period, and it declined rapidly to the pre-challenge level (Fig. 6e).

\section{Cytokine levels}

We measured IL-1 $\beta$, IL-4, IL-6, IL-8, IL-10, IL-12/ 23p40, IL-13, IL-17A, IFN- $\gamma$, and TNF- $\alpha$ in serum. The expression of the proinflammatory cytokines IL-6, IL-1 $\beta$ 

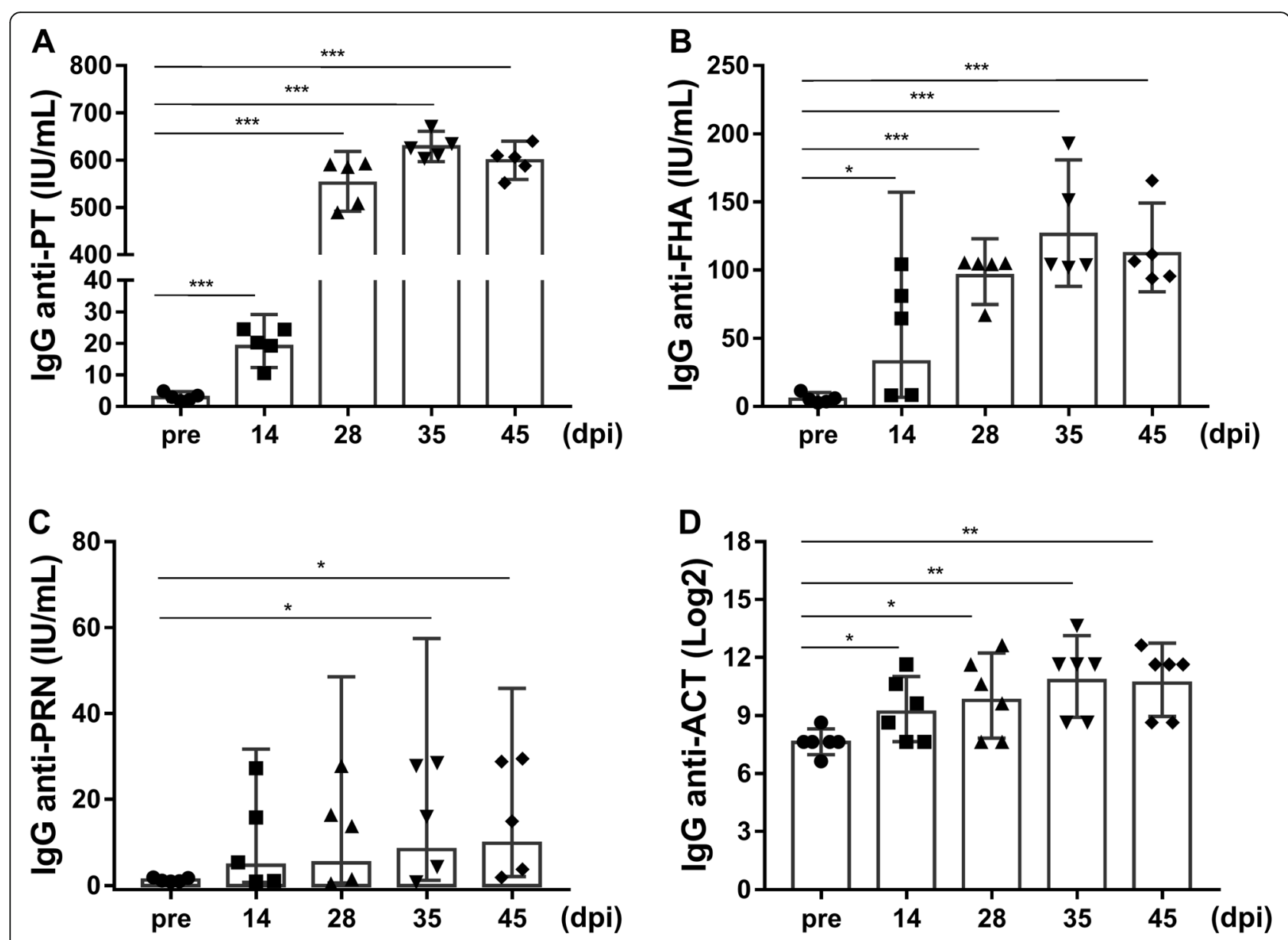

Fig. 3 Serological responses to PT, FHA, PRN and ACT in B.p-infected rhesus macaques. The results are presented as GMCs or GMTs and their $95 \%$ Cls. a Anti-PT. b Anti-FHA. c Anti-PRN. d Anti-ACT. ${ }^{*}, P<0.05,{ }^{* *}, P<0.01 ;{ }^{* * *}, P<0.001$ vs. pre-challenge; Student's t-test. The bars with error bars represent the geometric means with $95 \% \mathrm{Cls}(n=5)$

and TNF- $\alpha$ was significantly upregulated in all 7 macaques infected with strain 2016-CY-41(Fig. 7).

In addition, the increased IL-12/23p40 and IL-10 responses may suggest that $\mathrm{T}$ cell activation and regulation were increased in macaques after infection. And the time-dependent changes of these cytokines were presented in the additional file 4 . However, the expression of IL-4, IL-8, IL-13, IL-17A, and IFN- $\gamma$ did not exhibit significant changes.

\section{Discussion}

Pertussis is a vaccine-preventable childhood disease; however, there has been a resurgence in cases in recent years, including in countries with good vaccine immunization rates. A deep understanding of the immunology and epidemiology of this pathogen through studies on suitable experimental models, particularly NHP models, is important. Contrary to previous rhesus macaque challenge studies, in which most animals infected with pertussis have failed to develop obvious clinical manifestations of human pertussis $[10,14,20]$, the present study achieved the pertussis clinical spectrum in a rhesus macaque model. First, the characteristic whooping cough of pertussis syndrome, which has thus far been achieved only in a baboon model, was confirmed to have developed. All 5 challenged macaques and 2 macaques cohoused with the challenged macaques developed severe coughs that persisted for over 4 weeks. The coughing appeared on day 2 , peaked at day 15 , and decreased gradually thereafter. At peak illness, the coughing became severe, lasting $10-20 \mathrm{~s}$ per episode. Second, the number of CFUs in the NPW peaked at day 10 after challenge, reaching $6.2 \times 10^{6} \mathrm{CFU} / \mathrm{mL}$, and returned to baseline levels after 35 days. Third, a higher serum antibody responses of PT-IgG than of FHA-IgG was observed in serum. This is the same dynamic antibody responses as that observed in human pertussis. In humans, symptomatic pertussis is characterized by an elevated serum PT antibody response, while asymptomatic infection is characterized by an elevated serum FHA 


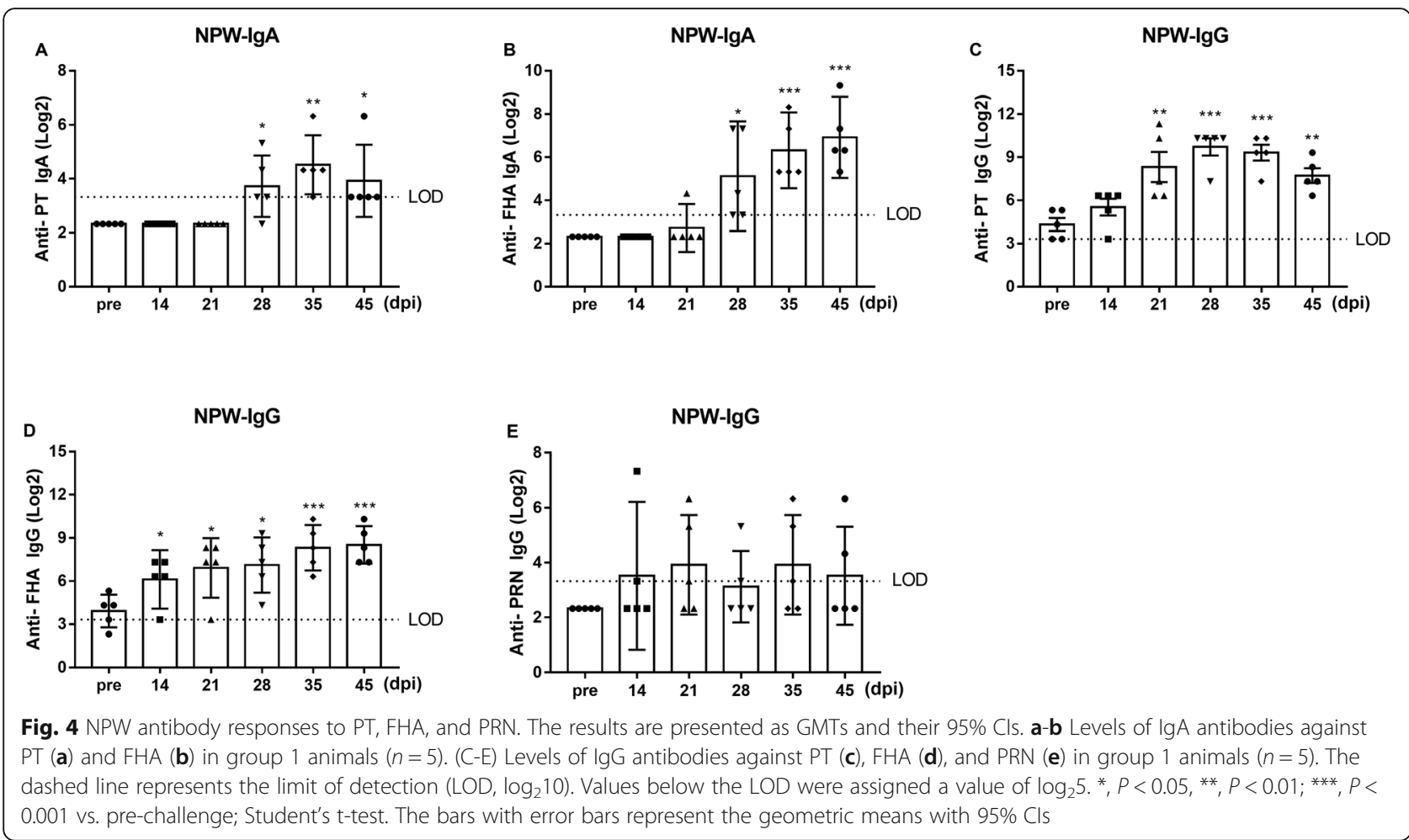

antibody response [21]. Moreover, the antibody responses in NPW also indicated that both anti-PT IgA and anti-FHA IgA levels were significantly higher than the pre-challenge levels; these changes are also observed in humans, and measurement of these antibodies has been used as a sensitive method for the diagnosis of pertussis [22, 23].

Although rhesus macaque models have been successfully established for several infectious diseases, the models for B.p have been insufficient [12-14]. Given the limited experimental details of the prior studies, which were performed in the 1920s-1950s, we speculated that the dose of infection, the method of infection, the age of the animal, and the infection strain may impact whether rhesus macaques can be successfully infected with B.p.

One of the most important factors for the pertussis model is the method of challenge. To date, nasal challenge, endotracheal intubation, in vivo injection, and aerosol challenge have been investigated in mouse models of pertussis $[24,25]$. In 1929, Sauer, L.W. et al. [12] reported that typical pertussis manifestations were observed in 3 of 10 rhesus macaques after intranasal infection and in 5 of 18 Cebus monkeys after intralaryngeal infection. In 1935, Culotta, C.S. et al. [13] reported that 1 of 16 rhesus macaques were successfully infected with B.p via the intratracheal route, while none of five macaques were successfully infected via the intranasal route. However, the B.p strains and infection doses were not mentioned in these studies. We previously infected 3 rhesus macaques ( $4-5$ years old) with strain 18,323 intranasally at a concentration of $5 \times 10^{8} \mathrm{CFU} / \mathrm{mL}$ in a 2 $\mathrm{mL}$ volume, but none of these animals developed a cough or leukocytosis. Three animals were assigned randomly to euthanasia for scheduled necropsies at 1,7 , and 14 dpi. No pathological changes were found in the lungs, tracheas, or lung-draining lymph nodes. We observed only very low levels of bacteria in the trachea at 1 and $7 \mathrm{dpi}$, and no bacteria were observed at $14 \mathrm{dpi}$. However, no lung colonization was observed at the three scheduled time points. Recently, aerosol challenge has been used as a novel challenge method to accurately simulate natural infections and reduce animal stress [26]. Using an aerosol apparatus, we previously challenged mice with different concentrations for different periods of time and successfully established a B.p infection mouse model [27]. In the present study, using the aerosol apparatus, we achieved the whole spectrum of symptoms of pertussis. Compared to other methods of experimental infection, aerosol challenge has great value because it accurately simulates natural infections, exhibits superior reproducibility and results in predictable distribution of infection and pathology $[28,29]$.

The dose of infection should be considered during model establishment. In a recent B.p challenge experiment on wP-vaccinated adults, pertussis colonization exhibited dose dependence. The dose was gradually escalated from $10^{3} \mathrm{CFU}\left(0 \%\right.$ colonized) to $10^{5} \mathrm{CFU}(80 \%$ colonized), while the minimum dose needed to induce 


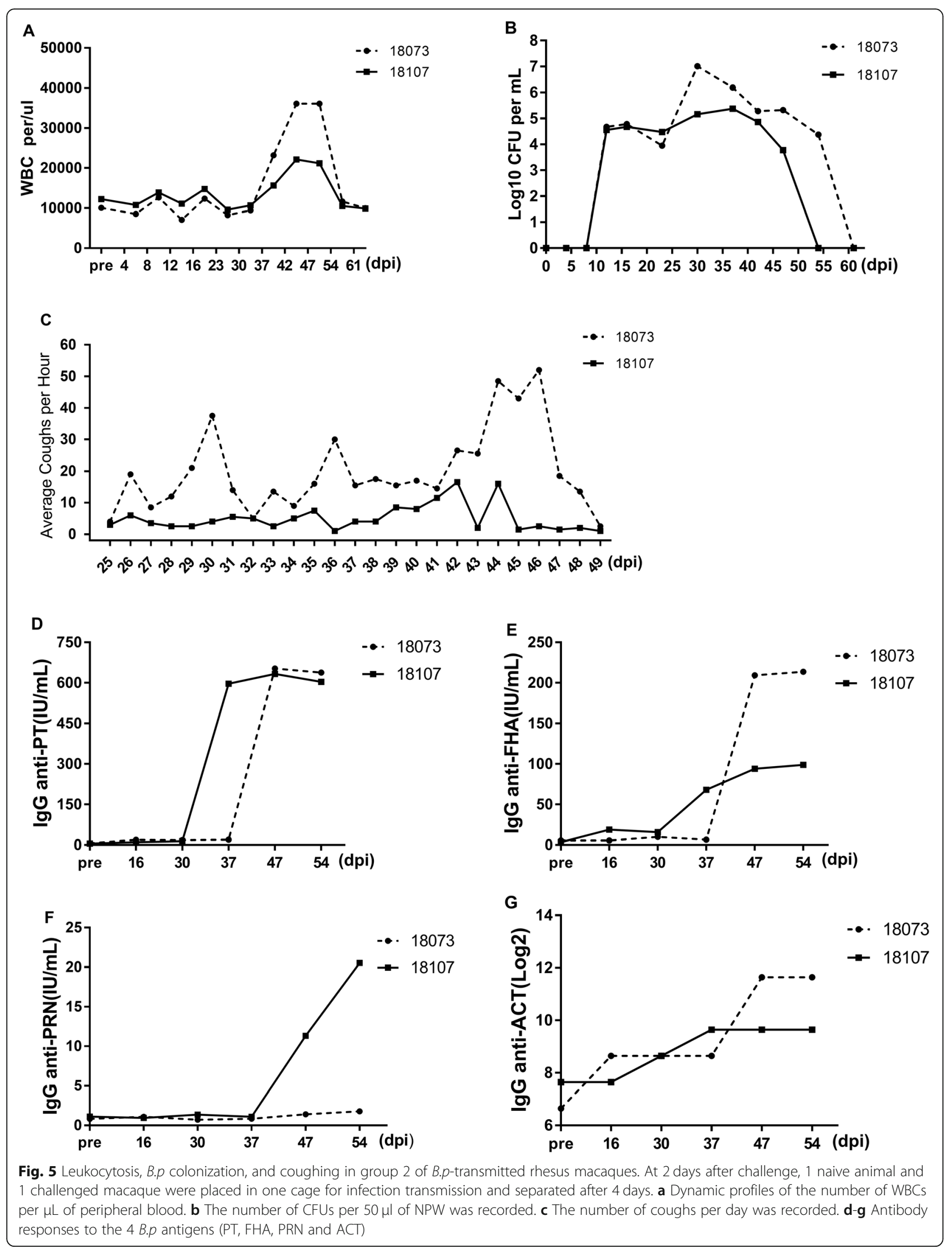




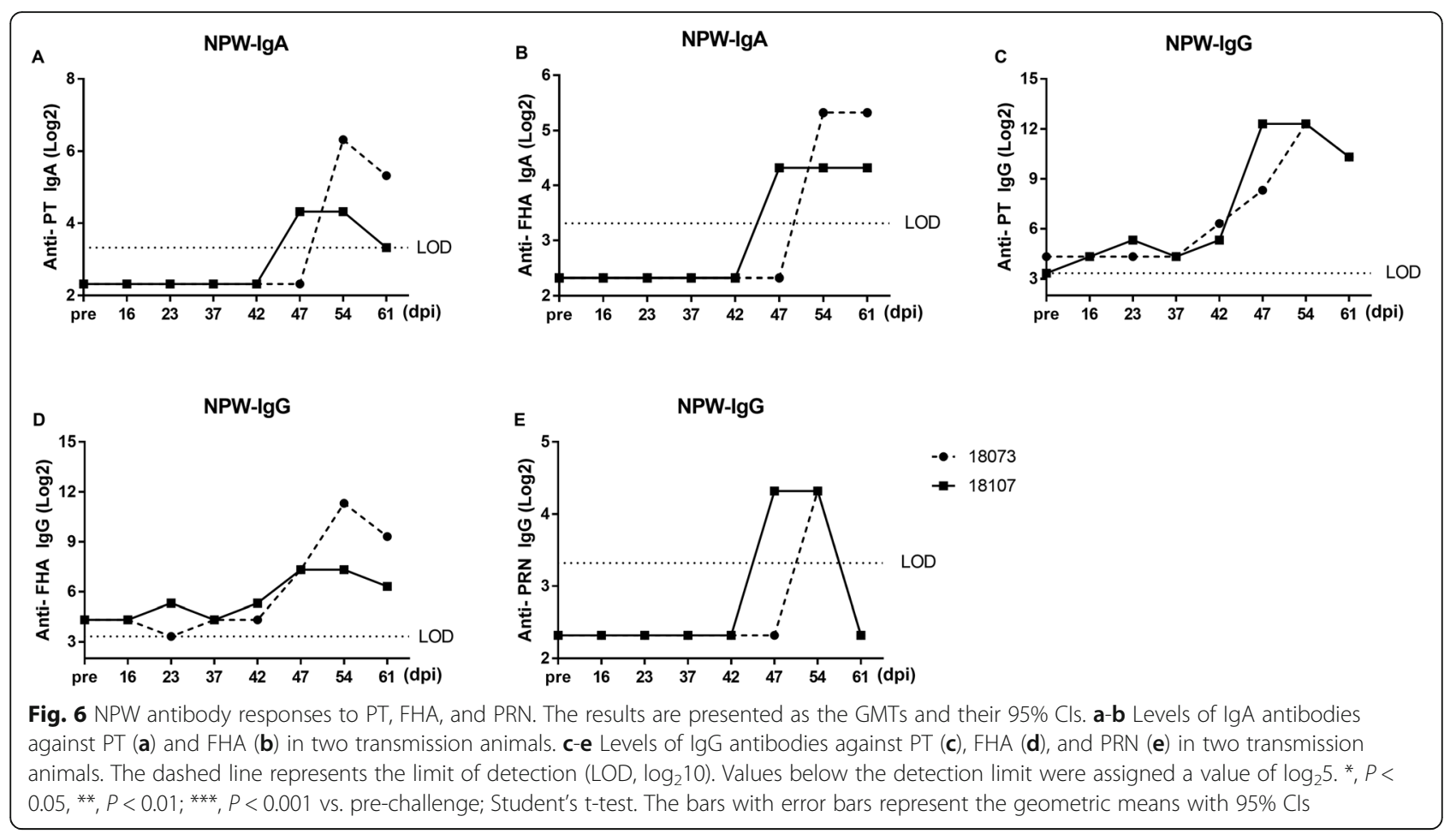

colonization was $10^{5} \mathrm{CFU}$. In 1940, North, E.A. et al. [14] infected rhesus macaques with $2 \mathrm{~mL}$ of $5 \times 10^{7}$ $\mathrm{CFU} / \mathrm{mL}$ B.p via the intranasal route or with $1 \mathrm{~mL}$ of 1 $\mathrm{mL} 5 \times 10^{7} \mathrm{CFU} / \mathrm{mL}$ B. $p$ via the intratracheal route; however, the resultant infection was mild, and spasmodic cough and protective antibodies were not observed. Warfel, J.M. et al. [10] intranasally infected 4 rhesus macaques ranging in weight from 1.7 to $1.9 \mathrm{~kg}$ and ranging in age from 43 to 64 weeks with $0.5 \mathrm{~mL}$ of $10^{9}$ to $10^{10}$ CFU/mL B.p; $50 \%$ of the monkeys developed significant increases in WBC count, and one of the two monkeys with increased WBC counts developed a mild cough. However, since the infectious B.p strain and the animal age were not clear in North, E.A. et al.'s study, we cannot comprehensively compare our findings with those of North, E.A. et al. In the present study, infant monkeys were challenged with a recently clinically isolated strain via an aerosol apparatus in which the bacterial concentration was maintained at $10^{4}-10^{5} \mathrm{CFU} / \mathrm{mL}$ for $60 \mathrm{~min}$. The constant dose of B.p during the challenge may be one reason for the success of the present infectious pertussis model.

Another critical factor to consider when establishing an animal model is the age of the animals. A previous study using an enterovirus type 71 (EV71) rhesus macaque model showed that, of the challenged animals, a clinical spectrum similar to that of humans was observed only in young animals [30]. Moreover, studies have demonstrated that young baboons show severe disease signs, whereas adult baboons show mild signs [10]. In one baboon pertussis model, 5- to 6-week-old baboons all developed fatal pertussis; however, in juvenile baboons, the infection was not fatal [31]. Frequently, asymptomatic adults have been implicated in the spread of infection to susceptible children [32]. In our study, 5- to 6month-old rhesus macaques were selected, and typical whooping cough, leukocytosis, bacteria-positive NPW, and transmission between animals were observed, similar to the results obtained in the baboon model. Therefore, we deduced that the challenge route as well as the age of the animals may influence B. $p$ infection.

The strain of bacteria used for challenge is another factor that should be noted. Strain 18,323 was used successfully to establish pertussis in $M$. cyclopis models in the 1960s; however, it could not induce overt signs of disease in rhesus macaques [16]. A recent analysis of the global population structure of B.p indicated that strain 18,323 (genotype ptxP4/ptxA5/prn6/fim2-2/fim3-1) belongs to a branch containing a small number of strains that are evolutionarily far from the major prevalent branch and revealed that strain 18,323 diverged from the prevalent strain branch approximately 2000 years ago [33]. Thus, in contrast to previous macaque animal studies, our study used a strain recently isolated from a clinic in China, 2016-CY-41 (genotype ptxP1/ptxA1/prn1/ fim2-1/fim3-1) (a common strain) and achieved the typical pertussis symptoms in infected macaques. Compared with that in the baboon model infected using strain D420 (genotype ptxP3/fim3-2), the peak symptom and disease progression in the macaque model infected 


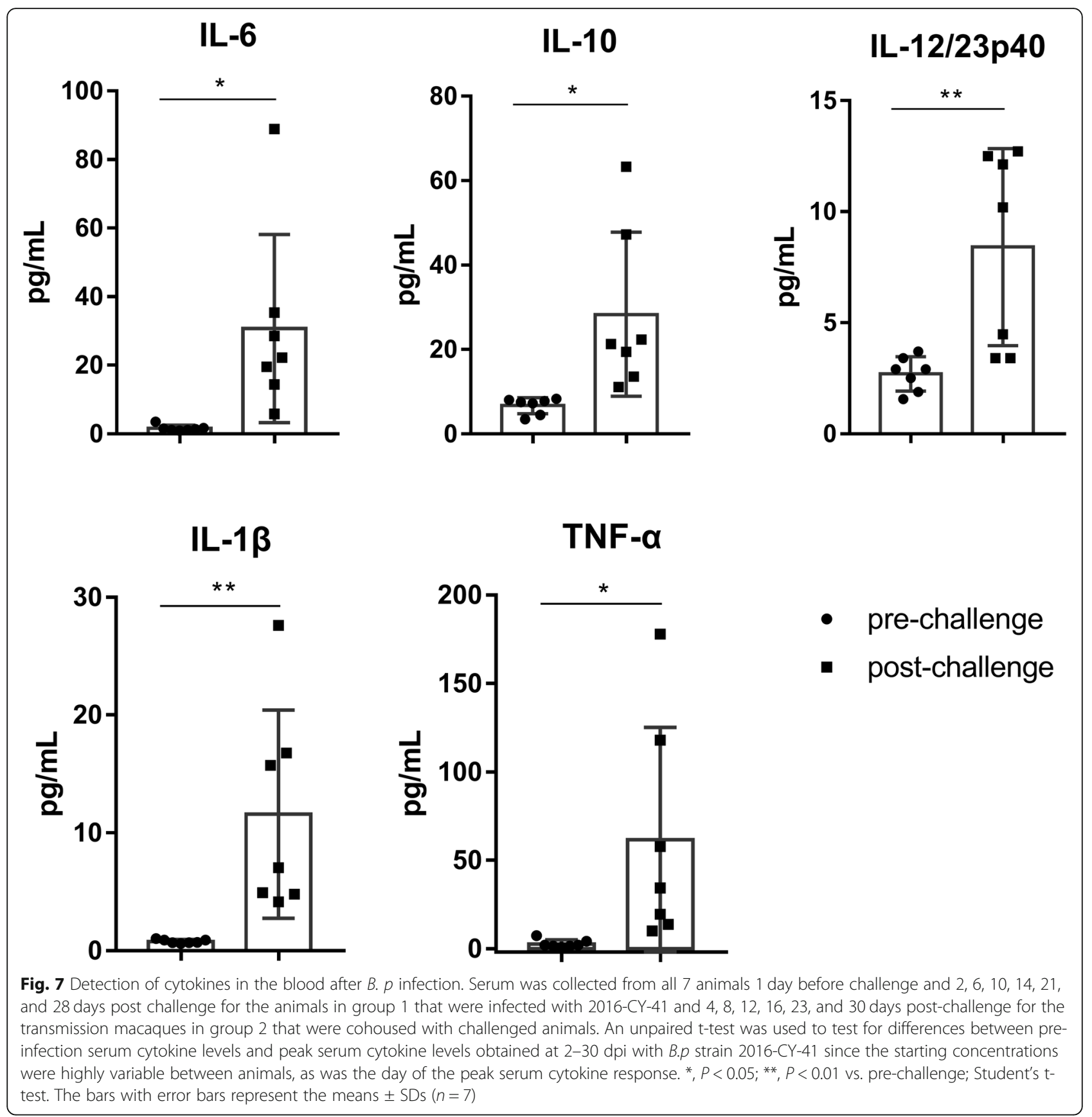

using B.p strain 2016-CY-41 was delayed [10]. The prevalence of ptxP3, one of the major components of D420, has increased in many European counties, the US, and Australia in the past 25 to 30 years, rather than that of ptxp1; however, in China, ptxP1 has remained predominant $[10,34,35]$. A SNP in ptxP3 that lies in a binding site for the transcriptional regulator BvgA may result in a strong promoter and increase the level of transcription of the associated PT [36]. Strains harbouring the ptxP3 allele have been found to be more virulent than ptxP1 strains in a mouse infection model, and they may also be associated with severe disease in humans [36-38]. Thus, we hypothesize that the genomic diversity of B.p may affect pertussis models. Further wholegenome sequencing experiments and analyses of virulence mechanisms and pertussis epidemiology should be performed in the future.

One of the possible reasons for the previous lack of success in establishment of rhesus macaque models of pertussis is high body temperature. The results of a temperature culture test in vitro showed that $\mathrm{ACT}$ protein levels are significantly lower in cells grown at $39^{\circ} \mathrm{C}$ 
than in cells grown at $37^{\circ} \mathrm{C}$, supporting the hypothesis that high temperature $\left(39^{\circ} \mathrm{C}\right)$ may result in loss of ACT expression, in turn resulting in a lack of B.p infection [10]. However, pathogenic microorganisms cultured in vitro may also exhibit virulence factor loss due to the lack of host selective pressures. During non-random culturing, B.p can undergo spontaneous phase variation involving multistep disappearance of virulence factors in the following order: ACT, PT and FHA [39]. The in vitro results suggest that the reduced expression of ACT is caused by the elevated normal body temperatures of rhesus macaques [10]. In the present in vivo study, we observed a 20 -fold increase in the anti-ACT antibody level, which was similar to the increase observed in the baboon model [40]. In addition, the rectal temperature was between $37.2^{\circ} \mathrm{C}$ and $39.9^{\circ} \mathrm{C}$ and did not exhibit significant changes. Thus, we deduce that body temperature may not be the only reason why previous rhesus macaque pertussis models have failed.

\section{Conclusion}

An infant rhesus macaque model of pertussis was established via aerosol challenge to provide a valuable alternative platform for research on pertussis pathogenesis and evaluation of vaccine candidates.

\section{Abbreviations}

B.p: Bordetella pertussis; NHP: Non-human primate; ptxP: Pertussis toxin promoter; ptxA: Pertussis toxin subunit; prn: Pertactin; NPW: Nasopharyngeal wash; dpi: Days post infection; WBC: White blood cell; FHA: Filamentous haemagglutinin; $A C T$ : Adenylate cyclase toxin

\section{Supplementary Information}

The online version contains supplementary material available at https:/doi. org/10.1186/s12879-021-06090-y.

Additional file 1: Additional Figure 1. Simplified layout of aerosol apparatus.

Additional file 2. Cough record of a rhesus macaques after aerosol challenge.

Additional file 3: Additional Figure 2. Rectal temperatures of rhesus macaques during the experiment.

Additional file 4: Additional Figure 3. Cytokines data obtained in the time-dependent manner.

\section{Acknowledgements}

We are grateful to the research staff members at the Department of Nonhuman Primate Research Center of the Institute of Medical Biology, Chinese Academy of Medical Sciences \& Peking Union Medical College, for their contribution to this research and to the Department of Diphtheria, Tetanus, and Pertussis Vaccine and Toxins of the National Institutes for Food and Drug Control for assisting with B.p strain 2016-CY-41.

\section{Authors' contributions}

LS and MS conceived and designed the study. WJ, CW, and DM performed the study. XM, LW, and PL provided the necessary reagents and performed serological testing. WZ, JL1, NG, and QG participated in the animal experiments. YM, JL2, and SL analyzed the data. LS, MS, and WJ wrote the manuscript. All authors read and approved the final manuscript.

\section{Funding}

This study was funded by the National Health and Family Planning Commission of China (2015ZX09101031), the Yunnan Provincial Science and Technology Department (2016GA004, 2016ZF003 and 2019HC006), and the CAMS Initiative for Innovative Medicine (2016-I2M-1-019). These funding agencies were not involved in the study design, data collection and analysis, decision to publish, or preparation of the manuscript.

\section{Availability of data and materials}

The datasets used and analyzed during the current study are available from the corresponding author on reasonable request.

\section{Declarations}

Ethics approval and consent to participate

The Committee on Ethics of the Institute of Medical Biology, Chinese Academy of Medical Sciences (IMBCAMS) approved the experiments with rhesus macaques in this study; the document identifer is DWSP201809002.

Consent for publication

Not applicable.

\section{Competing interests}

The authors declare that they have no competing interests.

\section{Author details}

${ }^{1}$ Institute of Medical Biology, Chinese Academy of Medical Science \& Peking Union Medical College, Kunming 650118, Yunnan, China. ${ }^{2}$ Yunnan Key Laboratory of Vaccine Research and Development on Severe Infectious Diseases, Kunming 650118, Yunnan, China. ${ }^{3}$ Department of Diphtheria, Tetanus and Pertussis Vaccine and Toxins, National Institute for Food and Drug Control, Beijing, China.

Received: 8 December 2020 Accepted: 20 April 2021

Published online: 03 May 2021

\section{References}

1. Cherry JD. The prevention of severe pertussis and pertussis deaths in young infants. Expert Rev Vaccines. 2019;18(3):205-8. https://doi.org/10.1080/14 760584.2019.1581065.

2. Paddock CD, Sanden GN, Cherry JD, Gal AA, Langston C, Tatti KM, et al. Pathology and pathogenesis of fatal Bordetella pertussis infection in infants. Clin Infect Dis. 2008;47(3):328-38. https://doi.org/10.1086/589753.

3. Barkoff AM, Mertsola J, Pierard D, Dalby T, Hoegh SV, Guillot S, et al. Pertactin-deficient Bordetella pertussis isolates: evidence of increased circulation in Europe, 1998 to 2015. Euro surveill. 2019;24(7):1700832. https:// doi.org/10.2807/1560-7917.ES.2019.24.7.1700832.

4. Pawloski LC, Queenan AM, Cassiday PK, Lynch AS, Harrison MJ, Shang W, et al. Prevalence and molecular characterization of pertactin-deficient Bordetella pertussis in the United States. Clin Vaccine Immunol. 2014;21(2): 119-25. https://doi.org/10.1128/CVI.00717-13.

5. Weigand MR, Pawloski LC, Peng Y, Ju H, Burroughs M, Cassiday PK, et al. Screening and genomic characterization of filamentous Hemagglutinindeficient Bordetella pertussis. Infect Immun. 2018;86(4):e00869-17.

6. Van Der Ark AA, Hozbor DF, Boog CJ, Metz B, Van den Dobbelsteen G, van Els CA. Resurgence of pertussis calls for re-evaluation of pertussis animal models. Expert Rev Vaccines. 2012;11(9):1121-37. https://doi.org/10.1586/ erv.12.83.

7. Elahi S, Holmstrom J, Gerdts V. The benefits of using diverse animal models for studying pertussis. Trends Microbiol. 2007;15(10):462-8. https://doi.org/1 0.1016/j.tim.2007.09.003.

8. Vandebriel RJ, Hellwig SM, Vermeulen JP, Hoekman JH, Dormans JA, Roholl PJ, et al. Association of Bordetella pertussis with host immune cells in the mouse lung. Microb Pathog. 2003;35(1):19-29. https://doi.org/10.1016/ S0882-4010(03)00087-1.

9. Safarchi A, Octavia S, Luu LD, Tay CY, Sintchenko V, Wood N, et al. Better colonisation of newly emerged Bordetella pertussis in the co-infection mouse model study. Vaccine. 2016;34(34):3967-71. https://doi.org/10.1016/j. vaccine.2016.06.052. 
10. Warfel JM, Beren J, Kelly VK, Lee G, Merkel TJ. Nonhuman primate model of pertussis. Infect Immun. 2012;80(4):1530-6. https://doi.org/10.1128/IAI.0631 $0-11$.

11. Melvin JA, Scheller EV, Miller JF, Cotter PA. Bordetella pertussis pathogenesis: current and future challenges. Nat Rev Microbiol. 2014;12(4):274-88. https:// doi.org/10.1038/nrmicro3235.

12. SAUER LW, HAMBRECHT L. Experimental whooping cough. Am J Dis Children. 1929;37(4):732-44.

13. Culotta CS, Harvey DF, Gordon EF. Whooping cough: II. Experimental study. J Pediatr. 1935;6(6):743-52. https://doi.org/10.1016/S0022-3476(35)80090-5.

14. North EA, Keogh EV, Christie R, Anderson G. Experimental pertussis in the monkey (MACACA MULATTA). Immunol Cell Biol. 1940;18(2):125-9. https:// doi.org/10.1038/icb.1940.13.

15. Lin T-m. Experimental whooping cough in monkey. J Formos Med Assoc. 1958;57(8):505-14.

16. Huang CC, Chen PM, Kuo JK, Chiu WH, Lin ST, Lin HS, et al. Experimental whooping cough. N Engl J Med. 1962;266(3):105-11. https://doi.org/10.1 056/NEJM196201182660301.

17. Vinson A, Prongay K, Ferguson B. The value of extended pedigrees for nextgeneration analysis of complex disease in the rhesus macaque. ILAR J. 2013; 54(2):91-105. https://doi.org/10.1093/ilar/ilt041.

18. van der Zee A, Schellekens JF, Mooi FR. Laboratory diagnosis of pertussis. Clin Microbiol Rev. 2015;28(4):1005-26. https://doi.org/10.1128/CMR.00031-1 5.

19. Wagner DK, Clements ML, Reimer CB, Snyder M, Nelson DL, Murphy BR Analysis of immunoglobulin $\mathrm{G}$ antibody responses after administration of live and inactivated influenza a vaccine indicates that nasal wash immunoglobulin G is a transudate from serum. J Clin Microbiol. 1987;25(3): 559-62. https://doi.org/10.1128/JCM.25.3.559-562.1987.

20. North EA, Keogh EV, Christie R, Anderson E. Experimental pertussis in the monkey. Aust J Exp Biol Med Sci. 1940;18(2):125-9. https://doi.org/10.1038/ icb.1940.13.

21. Long SS, Welkon CJ, Clark JL. Widespread silent transmission of pertussis in families: antibody correlates of infection and symptomatology. J Infect Dis. 1990;161(3):480-6. https://doi.org/10.1093/infdis/161.3.480.

22. Granström G, Askelöf $P$, Granström M. Specific immunoglobulin a to Bordetella pertussis antigens in mucosal secretion for rapid diagnosis of whooping cough. J Clin Microbiol. 1988;26(5):869-74. https://doi.org/10.112 8/JCM.26.5.869-874.1988.

23. Goodman YE, Wort AJ, Jackson FL. Enzyme-linked immunosorbent assay for detection of pertussis immunoglobulin a in nasopharyngeal secretions as an indicator of recent infection. J Clin Microbiol. 1981;13(2):286-92. https:// doi.org/10.1128/JCM.13.2.286-292.1981.

24. Kwon HJ, Han SB, Kim BR, Kang KR, Huh DH, Choi GS, et al. Assessment of safety and efficacy against Bordetella pertussis of a new tetanus-reduced dose diphtheria-acellular pertussis vaccine in a murine model. BMC Infect Dis. 2017;17(1):247-53. https://doi.org/10.1186/s12879-017-2369-x.

25. Wilk MM, Misiak A, McManus RM, Allen AC, Lynch MA, Mills KHG. Lung CD4 tissue-resident memory $T$ cells mediate adaptive immunity induced by previous infection of mice with bordetella pertussis. J Immunol. 2017;199(1): 233-43.

26. Sato Y, Izumiya K, Sato H, Cowell JL, Manclark CR. Aerosol infection of mice with Bordetella pertussis. Infect Immun. 1980;29(1):261-6. https://doi.org/1 0.1128/IAl.29.1.261-266.1980.

27. Mou D, Liang J, Gao N, Gu Q, Zhang M, Dai Y, et al. Aerosol challenge model in murine by Bordetella pertussis. J Postgrad Med. 2017;30(8):808-12.

28. Izumiya K. Aerosol infection of micewith Bordetella pertussis. Infect Immun. 1980;29(1):261-6.

29. Halperin SA, Heifetz SA, Kasina A. Experimental respiratory infection with Bordetella pertussis in mice: comparison of two methods. Clin Invest Med. 1988;11(4):297-303.

30. Zhao T, Zhang Z, Zhang Y, Feng M, Fan S, Wang L, et al. Dynamic interaction of Enterovirus 71 and dendritic cells in infected neonatal rhesus macaques. Front Cell Infect Microbiol. 2017;7:1-13.

31. Zimmerman LI, Papin JF, Warfel J, Wolf RF, Kosanke SD, Merkel TJ. Histopathology of bordetella pertussis in the baboon model. Infect Immun. 2018:86(11):e00511-18. https://doi.org/10.1128/IAl.00511-18

32. Althouse BM, Scarpino SV. Asymptomatic transmission and the resurgence of Bordetella pertussis. BMC Med. 2015;13(1):146. https://doi.org/10.1186/s12 916-015-0382-8.
33. Bart MJ, Harris SR, Advani A, Arakawa Y, Bottero D, Bouchez V, et al. Global population structure and evolution of bordetella pertussis and their relationship with vaccination. mBio. 2014;5(2):e01074-14.

34. Li L, Deng J, Ma X, Zhou K, Meng Q, Yuan L, et al. High prevalence of macrolide-resistant Bordetella pertussis and ptxP1 genotype, mainland China, 2014-2016. Emerg Infect Dis. 2019;25(12):2205-14. https://doi.org/1 $0.3201 /$ eid2512.181836

35. Xu Y, Zhang L, Tan Y, Wang L, Zhang S, Wang J. Genetic diversity and population dynamics of Bordetella pertussis in China between 1950-2007. Vaccine. 2015;33(46):6327-31. https://doi.org/10.1016/j.vaccine.2015.09.040.

36. Mooi FR, van Loo IH, van Gent M, He O, Bart MJ, Heuvelman KJ, et al. Bordetella pertussis strains with increased toxin production associated with pertussis resurgence. Emerg Infect Dis. 2009;15(8):1206-13. https://doi.org/1 0.3201/eid1508.081511

37. Clarke M, McIntyre PB, Blyth CC, Wood N, Octavia S, Sintchenko V, et al. The relationship between Bordetella pertussis genotype and clinical severity in Australian children with pertussis. J Infect. 2016;72(2):171-8. https://doi.org/1 0.1016/j.jinf.2015.11.004.

38. King AJ, Svd L, Mohangoo A, Mv G, Avd A, Bvd W. Genome-wide gene expression analysis of Bordetella pertussis isolates associated with a resurgence in pertussis: elucidation of factors involved in the increased fitness of epidemic strains. PLoS One. 2013:8(6):e66150. https://doi.org/10.13 71/journal.pone.0066150

39. Goldman S, Hanski E, Fish F. Spontaneous phase variation in Bordetella pertussis is a multistep non-random process. EMBO J. 1984;3(6):1353-6. https://doi.org/10.1002/j.1460-2075.1984.tb01976.x.

40. Eby JC, Gray MC, Warfel JM, Paddock CD, Jones TF, Day SR, et al. Quantification of the adenylate cyclase toxin of Bordetella pertussis in vitro and during respiratory infection. Infect Immun. 2013;81(5):1390-8. https:// doi.org/10.1128/IAI.00110-13

\section{Publisher's Note}

Springer Nature remains neutral with regard to jurisdictional claims in published maps and institutional affiliations.

\section{Ready to submit your research? Choose BMC and benefit from:}

- fast, convenient online submission

- thorough peer review by experienced researchers in your field

- rapid publication on acceptance

- support for research data, including large and complex data types

- gold Open Access which fosters wider collaboration and increased citations

- maximum visibility for your research: over $100 \mathrm{M}$ website views per year

At $\mathrm{BMC}$, research is always in progress.

Learn more biomedcentral.com/submission 